\title{
Evaluación de híbridos tri-lineales de maíz (Zea mays L.), por su reacción al Complejo Mancha de asfalto causada por los patógenos (Phyllachora maydis, Monographella maydis y Coniothyrium phyllacorae)
}

\author{
Héctor R. Deras F. ${ }^{1}$ - Reina Flor de Serrano ${ }^{2}$ - Carlos Mejía ${ }^{3}$ - Wenceslao Moreno ${ }^{4}$ \\ Centro Nacional de Tecnología Agropecuaria y Forestal, CENTA, El Salvador
}

1. Técnico investigador de maíz, hderasf@hotmail.com

2. Jefa de Laboratorio de Fitopatología, reinadeserrano@gmail.com

3. Asistente del programa de maíz

4. Técnico investigador región IV, wencesmor@yahoo.com

Recepción: 2014-04-24

Aceptación: 2014-05-24

\section{Resumen}

Durante 2013 se instaló un ensayo con el objetivo de identificar híbridos de maíz por su reacción a la enfermedad foliar conocida como Complejo Mancha de asfalto ${ }^{5}$. El ensayo se condujo en trece localidades de diferentes departamentos de El Salvador, teniendo nueve de ellas antecedentes de la enfermedad. La evaluación se desarrolló entre los meses de mayo a noviembre.

Se utilizó el diseño experimental alpha-látice $44^{\star} 5$, con tres repeticiones y 20 tratamientos. Los tratamientos se conformaron de 17 híbridos tri-lineales experimentales y tres híbridos comerciales como testigo. Se realizó un análisis de varianza por localidad y combinado, especialmente para porcentaje de mazorcas con mala cobertura; porcentaje de pudrición de mazorcas totales y porcentaje de mazorcas podridas afectadas por el complejo y rendimiento. Se utilizó el modelo AMMI Biplot GGE-SREG para determinar la interacción genotipo-ambiente, encontrándose significancia para dicha interacción; así como para los factores individuales en las que existió significancia al 1\% de probabilidad. En diez de las 13 localidades en la que se realizó el estudio, no existió presencia de la enfermedad. Pero en las restantes, la enfermedad incidió drásticamente sobre el rendimiento. El híbrido con mayor provecho en localidades sin presencia de la enfermedad fue DK-357 con 8.35 t ha-1, seguido por HES-3 y HES-1 con 7.75 y 7.1 t ha-1 respectivamente. En las localidades donde hubo presencia de la enfermedad, los mejores híbridos fueron: HES-3, HES-17 y DK-357, con rendimientos de $4.04,4.01$ y 4 t ha-1 respectivamente.

Los porcentajes de mazorcas afectadas por la enfermedad para estos híbridos fueron 50.3\%, 46.8\% y $60.7 \%$, respectivamente. El híbrido menos afectado por la enfermedad fue HES-1, con 42.9\% de mazorcas infectadas y rendimiento de 3.85 t ha-1. El análisis de estabilidad reportó como los híbridos más estables a HES-27 y HES-13. Los resultados obtenidos muestran a HES-1 como híbrido tolerante y a HES-3 como resistente.

Palabras clave: Maíz, Mancha de asfalto, híbrido, resistente, cultivo, enfermedad

\begin{abstract}
During the year 2013, a trial was installed with the objective to identify corn hybrids by its reaction to the foliar illness called Tar Spot Complex. The trial was conducted in thirteen places in different cities of El Salvador, and nine of them had antecedents with this illness. The evaluation was developed from May to November.

The experimental design Alpha-latice $4^{\star} 5$ was used, with three repetitions and 20 treatments. The treatments where made of 17 experimental tri-lineal hybrids and three commercial hybrids as witnesses. An analysis of variance was carried out by each place and combined, especially because of the percentage of cobs with bad coverage; percentage of rotting of the total of cobs and percentage of rotten cobs affected by the complex and performance. The AMMI Biplot GGE-SREG model was used to determine the genotype-environment interaction, finding significance for such interaction; as well as the individual factors that led to the $1 \%$ of probability. Ten out of the thirteen places where the study was carried out did not show the illness. But the other ones happened drastically upon the performance. The hybrid with higher advantage in the places with no presence of the illness was DK-357 with 8.35 ha-1, followed by HES-3 and HES-1 with 7.75 and 7.1 ha- 1 respectively. In the places where there was presence of the illness, the best hybrids were HES-3, HES-17 and DK-357, with performances of 4.04, 4.01 and $4 \mathrm{t}$ ha- 1 respectively.
\end{abstract}

The percentages of cobs affected by the illness for these hybrids were $50.3 \%, 46.8 \%$ and $60.7 \%$ respectively. The less affected hybrid by the illness was HES-1, with $42.9 \%$ of the infected cobs and performance of 3.85 t ha- 1 . The analysis of stability reported the more stable hybrids to HES-27 and HES-13. The results gathered show HES-1 as the tolerant hybrid and HES-3 as resistant.

Keywords: Corn, Tar Spot Complex, hybrid, resistant, farming, illness

5. De aquí en adelante, los autores también se referirán a ella mediante sus siglas CMA. 


\section{Introducción}

En El Salvador se cultivan alrededor de 406,089 manzanas de maíz con rendimiento promedio de $52 \mathrm{qq} / \mathrm{mz}$ (DGEA-MAG), siendo de los mayores rendimientos por unidad área de la región. El cultivo de maíz, junto con el de frijol, es la base de la alimentación de la familia salvadoreña.

El maíz, como el resto de granos básicos, se ha visto afectado por la incidencia de factores bióticos que ponen en peligro el rendimiento; y por ende, la seguridad alimentaria. A partir del período 2004 - 2005, la enfermedad foliar conocida como Mancha de asfalto tomó importancia económica para países como El Salvador, Guatemala, Honduras y Nicaragua; pues pasó de ser una enfermedad que aparece después de la etapa de elote duro -sin afectar el rendimiento-, a ser una enfermedad que aparece en las etapas de crecimiento vegetativo; afectando negativamente el rendimiento de grano.

Teóricamente, temperaturas altas durante el día y bajas durante la noche (17 a 22oC), con humedad relativa arriba del 75\%; fertilizaciones con exceso de nitrógeno y el excesivo monocultivo de maíz favorecen la enfermedad. En el país, la enfermedad se ha reportado entre los 300 a 600 metros sobre el nivel del mar. La Mancha de asfalto es causada -en primera instancia- por el hongo Phyllachora maydis. Si las condiciones ambientales son favorables para los patógenos, aparece un segundo hongo llamado Monographella maydis; luego aparece un tercer hongo llamado Coniothyrium phyllachorae. Estos tres hongos conforman lo que se denomina Complejo Mancha de asfalto.

Deras (s.f.), a partir de 2007, ha evaluado diversos germoplasma de maíz en búsqueda de resistencia genética para disminuir los efectos negativos causados por esta enfermedad. En 2011, el mismo investigador evalúa 51 híbridos triples blancos, identificando 17 materiales promisorios, los cuales forman parte de esta investigación.

\section{Materiales y métodos}

El desarrollo de la investigación se realizó durante los meses de mayo a noviembre, en diez localidades de la República con antecedentes de la enfermedad; $y$ en tres lugares sin reporte de la misma. La distribución de las localidades se muestra en la figura 1.

El manejo agronómico se efectuó según las recomendaciones de CENTA para el cultivo. El control de malezas se realizó en forma química con Atrazina 80 WP (2 kg ไha) más Alachlor (2lts \ha), ambas en forma manual. La fertilización fue realizada con fórmula 1515-15 (143 kg\ha), Urea (60 kg\ha) y Sulfato de amonio (143 $\mathrm{kg} \backslash \mathrm{ha}$ ). Las plagas del follaje se controlaron con Deltametrina 5\%, Thiacloprid Beta cyflutrin y Spinosad 2.5\% G. 


\section{Distribución de CMA en maíz en El Salvador}

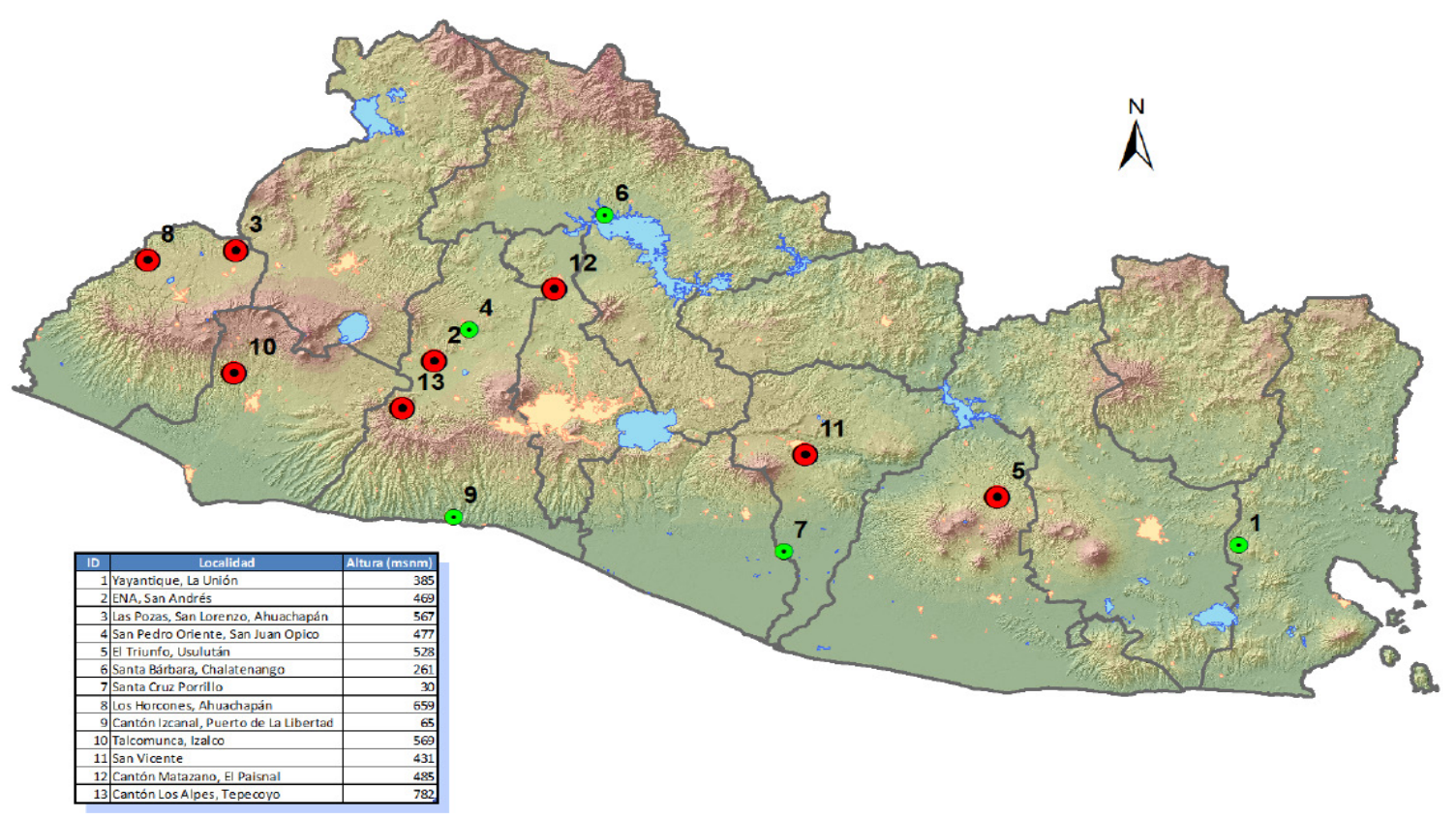

Figura 1. Distribución de ensayos para evaluación de Mancha de asfalto en El Salvador (2013).

Se utilizó el diseño experimental de alphalátice $5^{\star} 4$, con tres repeticiones y 20 tratamientos. Estos últimos se conformaron de 17 híbridos tri-lineales y tres híbridos comerciales como testigos. La unidad práctica y área útil se conformaron de dos surcos de cinco metros de largo y ochenta centímetros de distancia entre cada surco.

\section{Las variables estudiadas fueron:}

a. Cuantitativas. Días a floración masculina y femenina, altura de planta y mazorca, número de plantas y mazorcas cosechadas; porcentaje de acame de raíz, tallo, mazorcas podridas, mazorcas afectadas por la enfermedad y mala cobertura de mazorca; además de rendimiento de grano. b. Cualitativas. Enfermedades foliares, aspecto de planta y mazorca.

La evaluación de la incidencia de la enfermedad en el follaje se realizó en forma sistemática. Se hicieron dos lecturas entre 30-35 días y entre 70-75 días, después de siembra. Se seleccionó la octava planta del primer surco, y la dieciseisava del segundo. Se hicieron lecturas en la primera y tercer hoja de cada una durante el primer procedimiento. Mientras que en el segundo se analizó la hoja que envuelve a la mazorca y las dos hojas que se ubican debajo de esta.

También, se utilizó una escala de 1 a 5 para calificarlas, siendo 1 ausencia de la enfer- 
medad y 5 el follaje completamente dañado. Dicha escala es la sugerida por CIMMYT. Además, se realizó un análisis de varianza por localidad, un análisis combinado y uno de estabilidad, a través del modelo AMMI Biplot SCreg.

\section{Resultados y discusión}

\subsection{Efectos ambiente}

En la figura 2 se presentan los rendimientos obtenidos en cada una de las localidades, así como su coeficiente de variación. Se muestran además los porcentajes de cobertura de mazorcas y porcentaje de pudrición de las mismas.
El ensayo se efectuó en localidades con reportes de Mancha de asfalto; mientras que se descartaron otras que, por su altura sobre el nivel del mar, se estima que no existe presencia de la enfermedad. En tal sentido, Izcanal (departamento de La Libertad); Santa Bárbara (departamento de Chalatenango); Yayantique (departamento de La Unión); y Santa Cruz Porrillo (departamento de San Vicente) son las localidades donde no hay incidencia de CMA.

Los mayores rendimientos se obtuvieron en la localidad de San Cruz Porrillo, Tecoluca, con promedio de $8.87 \mathrm{t}$ ha-1 de diferencia es-

Cuadro resumen de evaluación de híbridos de maíz para tolerancia a Mancha de asfalto

\begin{tabular}{|lcccc|}
\hline \multicolumn{1}{|c}{ Localidad } & Rend t ha & $\begin{array}{c}\text { CV } \\
\text { (\%) }\end{array}$ & $\begin{array}{c}\text { Cobertura } \\
\text { mazorca } \\
(\%)\end{array}$ & $\begin{array}{c}\text { Pudrición } \\
\text { mazorca (\%) }\end{array}$ \\
\hline Pueblo Viejo (Ahuachapán) & $6.81^{* *}$ & 8.8 & 6.0 & 4.0 \\
Santa Bárbara (Chalatenango) & $4.64^{* *}$ & 9.4 & 4.4 & 7.6 \\
Cantón Matazano, El Paisnal (San Salvador) & $6.23^{* *}$ & 10.0 & 3.0 & 2.9 \\
Talcomunca, Izalco (Sonsonate) & 6.30 & 25.4 & 4.5 & 5.9 \\
Izcanal (La Libertad) & $6.15^{* *}$ & 10.8 & 7.3 & 9.0 \\
Pueblo Viejo (Ahuachapán) & 5.88 & 13.4 & 4.6 & 9.7 \\
Santa Cruz Porrillo, Tecoluca (San Vicente) & $8.87^{* *}$ & 6.3 & 7.9 & 7.2 \\
San Lorenzo (Ahuachapán) & 6.02 & 23.0 & 5.0 & 7.9 \\
San Vicente (San Vicente) & $7.24^{* *}$ & 11.0 & 8.8 & 6.9 \\
Yayantique (La Unión) & $7.83^{* *}$ & 15.0 & 3.3 & 3.4 \\
Cantón Las Palmas, Cantón El Triunfo & & & & \\
(Morazán) & $6.86^{* *}$ & 10.2 & & 4.5 \\
Los Alpes, Tepecoyo (La Libertad) & $2.73^{* *}$ & 23.7 & & 89.0 \\
San Andrés, La Libertad & $1.52^{* *}$ & 31.4 & & 90.6 \\
\hline
\end{tabular}

Figura 2. Análisis de varianza por localidad para las variables rendimiento de grano, porcentaje de mala cobertura y pudrición de mazorca, El Salvador (2013). 
tadística entre los híbridos. Contrariamente, la localidad con los menores rendimientos fue San Andrés con 1.52t ha-1 de diferencia estadística entre los híbridos. Cabe resaltar que, únicamente en tres de las doce localidades estudiadas no existió diferencia estadística entre los híbridos.

Los coeficientes de variación para esta variable oscilaron entre los 6.3 y 31.4\%. El bajo rendimiento obtenido en la localidad de Santa Bárbara en Chalatenango se debió al factor suelo; mientras que en San Juan Opico, Tepecoyo y San Andrés, el factor más determinante fue la incidencia del Complejo Mancha de asfalto, especialmente en Tepecoyo y San Andrés. Para el resto de localidades no hubo incidencia de la enfermedad. El reporte de comportamiento del rendimiento, porcentajes de mala cobertura y pudrición de mazorca en estas últimas localidades se realizó posteriormente; así como la incidencia o presencia del Complejo Mancha de asfalto.

Para la variable porcentaje de mala cobertura de mazorca, los mayores porcentajes correspondieron a la localidad de San Vicente con $8.89 \%$, mientras que el menor porcentaje correspondió a la localidad de Yayantique, La Unión (3.3\%). En general los porcentajes mostrados por los híbridos fueron menores a los máximos permitidos (10\%).

Para el porcentaje de pudrición de mazorcas, los mayores valores se presentaron en las lo- calidades de Tepecoyo y San Andrés con 89.0 y $90.68 \%$, respectivamente. Estos datos reflejan la incidencia del Complejo Mancha de asfalto. En las localidades de El Paisnal y Yayantique se obtuvieron menores porcentajes $(3.0 \%$ y $3.3 \%)$.

\subsection{Efecto genotipos}

En la figura 3 se muestra el análisis combinado para diez localidades, respecto a las variables de mayor interés: rendimiento de grano, porcentaje de mala cobertura de mazorca y porcentaje de mazorcas podridas.

Para el caso de rendimiento, el análisis reportó una alta diferencia estadística para los factores de localidades e híbridos; mientras que para la interacción híbrido por localidad diferencial, la cifra fue de 5\%. En tal sentido podemos afirmar que, aunque los lugares fueron diferentes unas de otras, esto influyó poco sobre los híbridos, los cuales respondieron de acuerdo a su carácter genético y en forma diferente.

El coeficiente de variación para el rendimiento fue de $14.3 \%$ con una media general de $6.60 \mathrm{t} \mathrm{h}-1$. El mayor rendimiento correspondió al testigo DK-357 con 8.35 t h-1; mientras que el menor fue para HES-11 con $4.30 \mathrm{th}-1$. (Figura 3). También es notable la diferencia de rendimiento existente entre el híbrido DK357 y el híbrido experimental HES-3 es de 0.6 t ha-1, la cual correspondió al 7\%. Por otra 
parte, el 55\% de los híbridos superaron la media general de rendimiento.

Para el porcentaje de mazorcas podridas, el análisis combinado reportó una alta diferencia entre los híbridos, correspondiendo el mayor porcentaje a DK-357 con 10.7\%. Esto fue ligeramente superior al máximo valor aceptado. Por el contrario, el menor porcentaje correspondió al híbrido experimental HES35 con $4.1 \%$. La diferencia del porcentaje de mazorcas podridas entre el híbrido de mayor rendimiento y mayor porcentaje de mazorcas podridas (DK-357), respecto al híbrido inmediato inferior en rendimiento (HES-3), fue de $5.2 \%$; siendo $6.5 \%$ la media general para esta variable. Es importante resaltar que estos porcentajes corresponden a mazorcas afectadas por enfermedades diferentes al Complejo Mancha de asfalto (Figura 3).

Respecto al porcentaje de mazorcas con mala cobertura, a excepción de dos híbridos experimentales (HES-31 y HES-21), el resto de ellos presentaron porcentajes menores al máximo permitido (10\%). En su caso, la media general fue de $5.5 \%$, como se puede apreciar en la figura 3.

Al evaluar la reacción de los híbridos frente al Complejo Mancha de asfalto en las diez localidades, en ningún de ellos se observó síntoma de la enfermedad. La evaluación de otras enfermedades se realizó utilizando la misma escala para Mancha de asfalto. El rango de daño por enfermedades osciló entre 2.3 a 3.0, sin que existiera mayor incidencia de enfermedades foliares.

En la localidad de San Pedro Oriente en San Juan Opico, La Libertad, el Complejo Mancha de asfalto se presentó levemente alrededor de los 85 a 90 días después de siembra, tiempo próximo a la etapa de secado o dobla (R5-R6). Sin embargo, el porcentaje de mazorcas podridas afectadas por la enfermedad mostraron todo lo contrario (Ver Anexo XX).

Para la localidad de Los Alpes en Tepecoyo, La Libertad, la afección del cultivo se presentó alrededor de los 70 a 75 días después de siembra, prácticamente en la etapa de elote (R2-R4), indiciendo drásticamente en el rendimiento. Situación similar se presentó en San Andrés, en donde el CMA se presentó entre los 40 a 45 días de después de siembra; es decir en la etapa de crecimiento vegetativo (V6). Esto influyó negativamente en el rendimiento (Figura 4).

La primera evaluación foliar para determinar la reacción de los híbridos a la enfermedad mostró a HES-1 como el combinado más tolerante, con una calificación de 1.0; mientras que DK-357 y HES-31 fueron los más susceptibles con calificación de 2.3. Para la segunda evaluación se mantuvo la tendencia de los materiales a la reacción de CMA; es decir, HES-1 presento una calificación de 1.2, mientras que DK-357 y HES-9 obtuvieron 
Cuadro resumen de características agronómicas de maíz para $\mathrm{CMA}^{6}$

\begin{tabular}{|c|c|c|c|c|c|c|c|}
\hline ENT & $\begin{array}{l}\text { Rend } \\
\text { t ha }\end{array}$ & $\begin{array}{c}\% \mathrm{Maz} \\
\text { Pod }\end{array}$ & $\begin{array}{c}\% \\
\text { Mala } \\
\text { Cober }\end{array}$ & Enf & $\begin{array}{r}\text { Phylla } \\
\text { chora }\end{array}$ & $\begin{array}{l}\text { Asp } \\
\text { Maz }\end{array}$ & $\begin{array}{l}\text { Asp } \\
\text { Pta }\end{array}$ \\
\hline DK-357 (20) & 8.35 & 10.7 & 7.3 & 2.5 & 1.0 & 2.6 & 2.8 \\
\hline HES-3(2) & 7.75 & 5.5 & 0.9 & 2.4 & 1.0 & 2.7 & 2.8 \\
\hline HES-1 (1) & 7.10 & 7.5 & 1.8 & 2.3 & 1.0 & 2.8 & 2.8 \\
\hline HES-9 (5) & 7.08 & 6.0 & 5.5 & 3.0 & 1.0 & 2.7 & 3.2 \\
\hline HES-27 (14) & 6.98 & 8.4 & 6.7 & 2.5 & 1.0 & 2.8 & 3.0 \\
\hline HES-31 (16) & 6.83 & 5.1 & 3.7 & 2.7 & 1.0 & 2.8 & 3.0 \\
\hline HES-13 (7) & 6.81 & 8.5 & 3.4 & 3.0 & 1.0 & 2.8 & 3.1 \\
\hline HES-17 (9) & 6.76 & 3.9 & 2.0 & 2.6 & 1.0 & 2.5 & 3.0 \\
\hline HES-31 (17) & 6.69 & 4.1 & 4.1 & 2.7 & 1.0 & 2.7 & 3.3 \\
\hline HES-5 (3) & 6.66 & 4.6 & 5.7 & 2.6 & 1.0 & 2.8 & 3.0 \\
\hline HES-29 (15) & 6.65 & 8.6 & 13.9 & 2.7 & 1.0 & 2.7 & 3.0 \\
\hline H-59 (18) & 6.59 & 7.7 & 7.4 & 2.7 & 1.0 & 2.8 & 3.2 \\
\hline HES-15 (8) & 6.52 & 5.1 & 6.6 & 2.7 & 1.0 & 2.6 & 3.0 \\
\hline HES-25 (13) & 6.48 & 7.9 & 2.9 & 2.7 & 1.0 & 3.0 & 3.0 \\
\hline HES-23 (12) & 6.40 & 4.9 & 2.6 & 2.9 & 1.0 & 3.0 & 3.2 \\
\hline HES-19 (10) & 6.33 & 5.2 & 7.4 & 2.4 & 1.0 & 2.7 & 3.0 \\
\hline PLATINO (19) & 5.99 & 6.6 & 3.2 & 2.7 & 1.0 & 2.8 & 3.0 \\
\hline HES-7(4) & 5.92 & 5.1 & 7.8 & 2.7 & 1.0 & 2.6 & 3.0 \\
\hline HES-21 (11) & 5.76 & 5.1 & 15.5 & 2.5 & 1.0 & 2.7 & 3.0 \\
\hline HES-11 (6) & 4.30 & 8.6 & 2.4 & 2.6 & 1.0 & 3.3 & 3.2 \\
\hline $\mathrm{X}$ & 6.60 & 6.5 & 5.5 & 2.6 & & 2.8 & 3.0 \\
\hline $\mathrm{CV}$ & 14.3 & 31.7 & 48.1 & 12.6 & & 12.6 & 10.6 \\
\hline LSD & 0.52 & 2.3 & 2.2 & $* *$ & & & ns \\
\hline Significancia (ent) & ** & ** & ** & & & & \\
\hline Significancia (loc*ent) & ** & * & ** & & & & \\
\hline
\end{tabular}

Figura 3. Principales características agronómicas de veinte híbridos de maíz por su reacción al complejo mancha de asfalto en diez localidades de El Salvador (2013).

6. Escala 1 a 5 (Siendo $1=$ Ausencia de la enfermedad; y 5= Follaje dañado o presencia de la enfermedad). 


\section{Cuadro resumen de reacción a CMA en tres localidades de El Salvador}

\begin{tabular}{|c|c|c|c|c|c|c|}
\hline ENT & $\begin{array}{c}\text { lera } \\
\text { lectura' }\end{array}$ & 2 da lectura ${ }^{1}$ & $\begin{array}{c}\text { 3era } \\
\text { lectura }{ }^{2}\end{array}$ & $\begin{array}{l}\text { Rend } \\
\text { t ha }^{-1}\end{array}$ & $\%$ Mz Pod & $\begin{array}{l}\% \mathrm{Mz} \\
\text { Phylla } \\
\text { chora }\end{array}$ \\
\hline HES-3 (2) & 2.1 & 3.2 & 3.2 & 4.04 & 46.2 & 50.3 \\
\hline HES-17 (9) & 2.2 & 2.8 & 2.3 & 4.01 & 46.8 & 46.8 \\
\hline DK-357 (20) & 2.3 & 3.7 & 3.8 & 4.00 & 66.1 & 60.7 \\
\hline HES-33 (17) & 2.0 & 3.3 & 3.3 & 3.93 & 63.3 & 60.2 \\
\hline HES-1 (1) & 1.0 & 1.2 & 1.0 & 3.85 & 54.4 & 42.9 \\
\hline HES-13 (7) & 2.2 & 3.2 & 2.5 & 3.67 & 61.5 & 51.8 \\
\hline HES-25 (13) & 2.2 & 3.3 & 2.8 & 3.54 & 56.9 & 48.9 \\
\hline HES-27 (14) & 2.2 & 3.4 & 3.2 & 3.49 & 67.3 & 62.9 \\
\hline HES-19 (10) & 2.2 & 2.9 & 2.3 & 3.39 & 66.8 & 63.2 \\
\hline HES-31 (16) & 2.3 & 3.5 & 3.7 & 3.39 & 66.3 & 55.6 \\
\hline HES-9 (5) & 2.2 & 3.6 & 3.5 & 3.39 & 69.6 & 51.3 \\
\hline PLATINO (19) & 2.0 & 3.2 & 3.2 & 3.22 & 67.4 & 44.5 \\
\hline $\mathrm{H}-59$ (18) & 2.1 & 3.2 & 3.2 & 3.13 & 67.0 & 63.4 \\
\hline HES-23 (12) & 2.0 & 2.3 & 2.3 & 3.07 & 62.1 & 68.6 \\
\hline HES-15 (8) & 2.0 & 3.0 & 3.2 & 3.06 & 64.6 & 60.0 \\
\hline HES-29 (15) & 2.0 & 3.0 & 2.7 & 3.05 & 65.4 & 54.7 \\
\hline HES-5 (3) & 2.2 & 3.3 & 3.3 & 3.00 & 67.7 & 69.7 \\
\hline HES-7 (4) & 2.0 & 2.8 & 3.0 & 2.93 & 65.6 & 60.5 \\
\hline HES-21 (11) & 1.9 & 2.7 & 2.5 & 2.80 & 67.0 & 67.0 \\
\hline HES-11 (6) & 1.7 & 2.5 & 2.5 & 2.58 & 70.4 & 55.6 \\
\hline $\mathrm{X}$ & 2.08 & 3.00 & 2.9 & 3.37 & 63.1 & 56.9 \\
\hline $\mathrm{CV}$ & 14.0 & 15.2 & 18.4 & 21.09 & 16.2 & 39.3 \\
\hline $\operatorname{LSD}(0.05)$ & 0.33 & 0.5 & 0.5 & 0.66 & 9.5 & 20.9 \\
\hline Significancia (hib) & ** & ** & ** & ** & ** & * \\
\hline Significancia (loc'hib) & ** & ** & ** & ** & ** & ** \\
\hline
\end{tabular}

Figura 4. Principales características agronómicas de 20 híbridos de maíz frente a CMA (2013).

una calificación de 3.7 y 3.6, respectivamen- del follaje, resultando HES-1 como el híbrido te. Estos resultados correspondieron a las lo- con menor presencia de la enfermedad (1.2 de calidades de San Andrés y Tepecoyo. En este calificación); siendo DK-357 y HES-31 los más último sitio se realizó una tercera evaluación susceptible (3.8 y 3.7 de calificación respectiva). 
Al comparar el rendimiento entre las diez localidades con ausencia de la enfermedad y los tres sitios infectados, los rendimientos en forma general disminuyeron en un 51\% para estos tres últimos. La media general para las tres localidades fue de $3.37 \mathrm{t}$ ha-1, correspondiendo el mayor rendimiento a HES-3 con 4.04 tha-1; y el menor rendimiento a HES-11 con $2.58 \mathrm{t}$ ha-1. Por su parte, DK-357 ocupó el segundo lugar con 4.01t ha-1 en las tres localidades. Es interesante que HES-3 ocupara el segundo lugar en las localidades donde no existió la presión de la enfermedad.

Para determinar en qué medida el Complejo Mancha de asfalto incidió sobre la calidad del cultivo, no solo se determinó el porcentaje total de mazorcas podridas, sino también, se tipificó qué porcentaje de ellas se encontraban así debido a la enfermedad. En ese sentido se analizan los tres híbridos superiores en rendimiento, comparándose ambos porcentajes.
HES-3 reportó un 46.2\% de mazorcas podridas totalmente, de las cuales el 50.3\% correspondió al Complejo Mancha de asfalto. HES17 mostró un 46.8\% de mazorcas podridas debido al Complejo. DK-357 reportó obtuvo un total de $66.1 \%$, siendo el $60.7 \%$ de ellas infectadas por CMA. En forma general, el híbrido más afectado por el Complejo Mancha de asfalto fue HES-5 con $69.7 \%$ de mazorcas podridas, en comparación con el híbrido HES1 (42.9\%). En segundo lugar se encontró el testigo Platino con $44.5 \%$. La media general para porcentajes de mazorcas afectadas por la enfermedad fue $56.9 \%$, determinándose una diferencia estadística entre las muestras, siendo unas más susceptibles que otras.

\subsection{Interacción genotipo por ambiente}

A continuación se presenta el análisis AMMI Bi plot para la variable rendimiento de grano a través de las diez localidades.

El modelo AMMI Bi plot determinó una significancia para la interacción genotipo-

Cuadro resumen para variable de rendimiento de grano de maíz

\begin{tabular}{lccccc}
\hline Fuente de V & g.l. & Tipo III SS & C Medio & F-Valor & Pr $>$ F \\
\hline loc & 9 & 735.19 & 81.69 & 24.28 & $<.0001$ \\
rep(loc) & 20 & 67.28 & 3.36 & 3.80 & $<.0001$ \\
trat & 19 & 337.63 & 17.77 & 20.08 & $<.0001$ \\
loc & 171 & 208.51 & 1.22 & 1.38 & 0.0059 \\
\hline
\end{tabular}

Figura 5. Análisis de varianza para rendimiento de grano de 20 híbridos de maíz evaluados a través de diez localidades en El Salvador (2013). 
ambiente, mientras que para los factores individuales de localidad e híbrido -en el que existieron diferencias altamente significativas-, existió un resultado similar con el análisis combinado presentado en el apartado 3.2.
Para determinar el híbrido más estable se consideró el valor de PCA 2 más cercano a cero, que estuviera sobre el promedio general; siendo para este caso $6.6 \mathrm{t}$ ha-1.

\section{Cuadro resumen de las puntuaciones AMMI para híbridos de maíz evaluados}

\begin{tabular}{lcrr}
\hline Híbrido & REND & \multicolumn{1}{l}{ PCA1 } & \multicolumn{1}{c}{ PCA2 } \\
\hline HES-1 & 7.07 & 0.4951 & 0.1525 \\
HES-3 & 7.84 & 1.2662 & -0.4699 \\
HES-5 & 6.51 & -0.0957 & -0.1030 \\
HES-7 & 6.07 & -0.4848 & -0.4207 \\
HES-9 & 6.92 & 0.2132 & 0.2822 \\
HES-11 & 4.45 & -2.1255 & -0.6858 \\
HES-13 & 6.77 & 0.1628 & 0.0582 \\
HES-15 & 6.59 & 0.0779 & -0.4059 \\
HES-17 & 6.83 & 0.2068 & 0.1740 \\
HES-19 & 6.43 & -0.1489 & -0.3742 \\
HES-21 & 5.90 & -0.8029 & 0.7285 \\
HES-23 & 6.27 & -0.2985 & 0.0829 \\
HES-25 & 6.48 & -0.0273 & -0.7391 \\
HES-27 & 6.92 & 0.2776 & -0.0043 \\
HES-29 & 6.62 & 0.0127 & 0.6964 \\
HES-31 & 6.85 & 0.2260 & 0.1228 \\
HES-33 & 6.62 & -0.0293 & 0.7743 \\
H-59 & 6.51 & 0.0552 & -0.1839 \\
PLATINO & 5.93 & -0.7327 & 0.5018 \\
DK-357 & 8.37 & 1.7520 & -0.1867 \\
\hline
\end{tabular}

Figura 6. Puntuaciones o valores AMMI para los híbridos de las diez localidades estudiadas, El Salvador (2013). 
Según los valores AMMI los híbridos más estables serían HES-27 y HES-13 y con rendimientos arriba de la media general. Considerando que la diferencia entre la estabilidad no es muy amplia, reacción al Complejo Mancha de asfalto y el potencial de rendimiento, se afirma que HES-1 y HES-3 son híbridos con buenas perspectivas. Es importante resaltar que estos híbridos fueron los mejores en la evaluación realizadas en 2011.

Respecto a los testigos utilizados, el mejor de ellos en cuanto a rendimiento fue DK-357, seguido por H-59 y Platino. Sin embargo, Platino reacciona mejor contra el Complejo Mancha de asfalto, a pesar de que su rendimiento fue afectado por la baja población en todas las localidades.

\section{Conclusiones}

Bajo las condiciones en que se desarrolló la evaluación se concluye lo siguiente:

En distintos lugares del departamento de La Libertad como San Pedro Oriente, la enfermedad se presentó en la etapa de secamiento, afectando medianamente el rendimiento; mientras que en Los Alpes, el CMA se afectó durante la etapa de elote. No obstante, en San Andrés el rendimiento se redujo casi por completo. Todo esto confirma que el daño de la afección depende de la etapa del cultivo en que se presente.
En las localidades sin presión de la enfermedad, los mayores rendimientos se obtuvieron con DK-357, HES-3 y HES-1 con 8.35, 7.75, 7.10t ha- 1 ; siendo $10.7 \%$, $5.5 \%$ y $7.5 \%$ los porcentajes de pudrición de mazorca, respectivamente.

En las localidades con presión de la enfermedad, los híbridos con mayor rendimiento fueron HES-3, HES-17, DK-357 con 4.04, 4.01, 4.0t ha-1 con porcentaje respectivo de mazorcas afectadas de $50.3 \%$, $46.8 \%$ y $60.7 \%$.

El combinado más afectado por Mancha de asfalto fue HES-5 con $69.7 \%$ de mazorcas enfermas. Contrariamente, los híbridos menos afectados fueron HES-1 y Platino con 42.9 y $44.5 \%$.

De acuerdo al análisis de estabilidad, los híbridos más estables fueron HES-27 y HES-13.

Basados en los resultados obtenidos, se afirma que los híbridos HES-3 y HES-17 son tolerantes al Complejo Mancha de asfalto, mientras que HES-1 es resistente.

Como puntos finales del estudio se recomienda evaluar en campo de agricultores los híbridos HES-1 y HES-3, junto con otros germoplasmas de atributos específicos, a fin de valorizar su continuidad. Asimismo, se sugiere realizar pruebas de coincidencia de los híbridos antes mencionados, para determinar la factibilidad de producción de semilla en sus distintas categorías. 


\section{Referencias bibliográficas}

Agrios, G. N. (1989). Fitopatología. Editorial Limusa. Tercera reimpresión. México.

Deras F., Héctor R. (s.f.). Evaluación de híbridos blancos de maíz (Zea mays) tri-lineales con resistencia y/o tolerancia relativa al Complejo Mancha de asfalto (Phyllachora maydis, Monographela maydis). Sin publicar.

Jugenheimer, R. W. (1988). Maíz. Variedades mejoradas, métodos de cultivo y producción de semillas. Editorial Limusa. Tercera reimpresión. México.

Programa de maíz del CIMMYT (2004). Enfermedades del maíz: Una guía para su identificación en el campo. Cuarta edición. México, D.F. CIMMYT 